\title{
Rototaxis: Localization of active motion under rotation
}

\author{
Yuanjian Zheng $\oplus^{1,2}$ and Hartmut Löwen $\oplus^{2}$ \\ ${ }^{1}$ Division of Physics and Applied Physics, School of Physical and Mathematical Sciences, Nanyang Technological University, Singapore \\ ${ }^{2}$ Institut für Theoretische Physik II: Weiche Materie, Heinrich-Heine-Universität Düsseldorf, D-40225 Düsseldorf, Germany
}

(Received 9 December 2019; accepted 30 March 2020; published 27 April 2020)

\begin{abstract}
The ability to navigate in complex, inhomogeneous environments is fundamental to survival at all length scales, giving rise to the rapid development of various subfields in biolocomotion such as the well established concept of chemotaxis. In this work, we extend this existing notion of taxis to rotating environments and introduce the idea of "rototaxis" to biolocomotion. In particular, we explore both overdamped and inertial dynamics of a model synthetic self-propelled particle in the presence of constant global rotation, focusing on the particle's ability to localize near a rotation center as a survival strategy. We find that, in the overdamped regime, a torque directing the swim orientation to the rotation origin enables the swimmer to remain on stable epicyclical-like trajectories. On the other hand, for underdamped motion with inertial effects, the intricate competition between self-propulsion and inertial forces, in conjunction with the rotation-induced torque, leads to complex dynamical behavior with nontrivial phase space of initial conditions which we reveal by numerical simulations. Our results are relevant for a wide range of setups, from vibrated granular matter on turntables to microorganisms or animals swimming near swirls or vortices.
\end{abstract}

DOI: 10.1103/PhysRevResearch.2.023079

\section{INTRODUCTION}

Microorganisms and artificial microswimmers alike [1-3], are highly capable of adapting their motion in response to gradients present in external stimuli. This inherent property of life, often collectively referred to as taxis [4], is not only extremely crucial to survival and the emergent evolutionary fitness of living species, but also underpins the complex dynamics of artificial colloidal particles that are showing increasing promise for a wide range of industrial applications [5]. Such complex behavior of microswimmers emerges in response to many forms of physical stimuli such as variation in the spatiotemporal distribution of chemical concentrations (chemotaxis) [6-14], the intensity of light (phototaxis) [15-25], external magnetic (magnetotaxis) [26-31] or gravitational field strengths (gravitaxis) [32-37], temperature (thermotaxis) [38-40], viscosity (viscotaxis) [41], orientation of the solvent flow field (rheotaxis) [42-44], and even for topographical gradients that may be present in distorted solids or environments (topotaxis) [45-48].

In this work, we consider a form of motion in response to an external stimulus that takes the form of a gradient in the local kinetic energy of the surrounding flowing fluid. For a globally rotating environment, this form of dynamics has several advantages as a survival strategy [49-51]. First, a propensity for the positive gradient which we term positive rototaxis, corresponding to the drive towards a region where

Published by the American Physical Society under the terms of the Creative Commons Attribution 4.0 International license. Further distribution of this work must maintain attribution to the author(s) and the published article's title, journal citation, and DOI. solvent flow is large, would be an ideal strategy for enhanced transport in situations where an organism seeks to escape from death or destruction in vortex environments such as whirlpools or maelstroms, or where it is attempting to chase down a prey [52]. Conversely, negative rototaxis corresponds to the localization of dynamical trajectories in the vicinity of rotation centers and would thus be largely favorable for remaining near static food sources, maintaining proximity to swarm centers [53], or simply energy saving by not swimming against the flow in low vorticity scenarios. In this context, we note that ideal rototaxis occurs when the swim orientation perfectly aligns along the gradient of kinetic energy $\nabla \vec{u}^{2}(\vec{r})$, where $\vec{u}(\vec{r})$ is the flow velocity. This should be contrasted to ordinary rheotaxis [42-44], where the alignment of the swim axis is along the flow direction $\vec{u}(\vec{r})$ itself.

Despite its survival advantages as a taxis strategy, generating negative rototaxis is a considerably difficult task for microswimmers. This is due to the centrifugal force in the rotating frame of the system that tends to expel the particle or organism away from its rotation center. In this work, we introduce a model 2D microswimmer capable of generating a tunable torque that sustains both negative and positive rototaxis by simply altering its initial swim orientation with respect to its position in the flow field. This is made possible in the presence of inertia that is increasingly being recognized to play a pivotal role in the dynamics of microswimmers [54-58].

This paper is organized as follows. In Sec. II, we model the basic physics and equations of motion for an active microswimmer in a rotating, noise-free environment. This minimal bead model is designed to capture the essential features of a large class of rod-shaped axial propelled bacteria such as well-studied model organisms Escherichia coli and Bacillus subtilis [59], while still allowing for modular generalizations 
by attachment or removal of additional beads or structures for more complex geometries and swim orientation. Moreover, synthetic realizations of such bead models [50,60-67] in the overdamped limit has also recently been achieved [68], giving way for potential experimental verification of our results. We then solve these equations in the overdamped regime in Sec. III. Within a linear stability analysis, we show that this results in stable epicycliclike trajectories and rosettes in both the rest and rotating frames of real space. We then numerically solve the nonlinear inertial problem in Sec. IV to reveal the existence of both positive and negative rototactic phases that is intricately dependent on initial conditions. In addition, we also find a further differentiation in the negative rototactic phase: the emergence of circular periodic limit cycles in addition to the existing epicyclic and rosette solutions found in the overdamped limit. Lastly, in Sec. V, we discuss the implications of our work and draw conclusions.

\section{MODEL}

We begin by considering a single particle consisting of two beads that are point masses, $m_{1}$ and $m_{2}$ (i.e., the total mass of the particle is $\left.m=m_{1}+m_{2}\right)$ at positions $\vec{r}_{1}$ and $\vec{r}_{2}$ connected by a light inextensible rod of length $l \equiv\left|\vec{r}_{1}-\vec{r}_{2}\right|$. The particle is confined to a $2 \mathrm{D}$ plane and is acting under a global rotation of angular frequency $\vec{\omega}$ about some fixed origin in the plane, corresponding to an effective flow field $\vec{u}(\vec{r})=\vec{\omega} \times \vec{R}$.

Activity is introduced to the model microswimmer by a persistent self-propulsion velocity $v_{0} \hat{n}$ projecting from the geometrical center of the two beads $\vec{R}$ in a direction along the long axis of the particle towards $\vec{r}_{2}$ such that each bead is always $l / 2$ away from $\vec{R}$, where $\hat{n} \equiv\left(\vec{r}_{2}-\vec{r}_{1}\right) / l=$ $[\cos \phi, \sin \phi]^{T}$ and $\phi(t)$ is the swim orientation phase angle, defined with respect to the rest frame of the system from some positive $x$ axis.

Frictional effects arising from translational $(\gamma)$ and rotational $\left(\gamma_{R}\right)$ viscosities are assumed to be acting on the geometrical center $\vec{R}$ and orientational $\phi$ degrees of freedom, and are also assumed to be linearly proportional to the translational and angular velocities in the rotating frame, respectively. The translational and swim orientational equations of motion (in the rest frame) are thus written as

$$
\begin{aligned}
& m \ddot{\vec{R}}+\gamma(\dot{\vec{R}}-\vec{\omega} \times \vec{R})=\gamma v_{0} \hat{n}+\vec{f}(t), \\
& J \ddot{\phi}+\gamma_{R}(\dot{\phi}-\omega)=M+\tau(t)+g(t),
\end{aligned}
$$

where the translational degree of freedom is the geometrical center $\vec{R}=\vec{r}_{i}+\frac{(3-2 i) l}{2} \hat{n}$ for $(i=1,2)$ and where $J=\sum_{i} m_{i}\left(\vec{R}-\vec{r}_{i}\right)^{2}=\frac{m}{4} l^{2}$ is the moment of inertia of the dumbbell-like particle. For full generality and completeness, we also include contributions from fluctuating forces $\vec{f}(t)$ and torques $\vec{g}(t)$ from the solvent that are typically modeled as Gaussian white noise acting on $\vec{R}$ and $\phi$, respectively [2], as well as provision for an internal torque $M$ that allows for the modeling of circle swimmers [69]. However, for simplicity, $f(t), g(t)$, and $M$ are all assumed to be zero in our model of rototaxis hereinafter.

The term $\tau(t)$ controls rototaxis, and is referred to as the rotation-induced torque. Here this "rototactic" torque is a con-
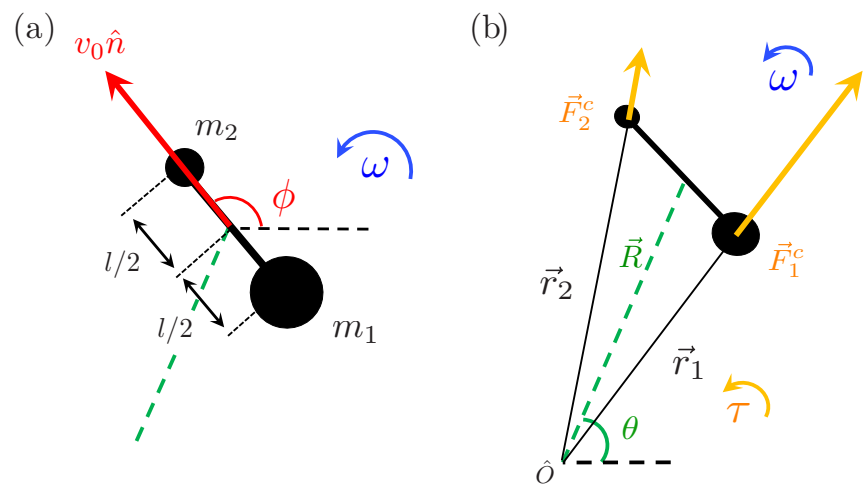

FIG. 1. Schematics of the rototactic microswimmer. (a) Selfpropulsion (red) with swim orientation $\phi$ of an axial swimmer under a constant global rotation $\omega$ (blue). (b) Positional degrees of freedom $R$ and $\theta$ defined with respect to the same axis as $\phi$ from the center of rotation $\hat{O}$. Torque (orange) generated from centrifugal forces $\vec{F}_{i}^{c}$ that may in general be opposing the global rotation $\omega$.

sequence of the centrifugal forces acting on the dumbell-like swimmer. To calculate it, the inertial torque $\tau^{\prime}(t)$ experienced by the particle in the rotating frame is identical to $\tau(t)$ in the rest frame (i.e., $\tau^{\prime}=\tau$ ), and originates from fictitious centrifugal $\vec{F}_{c}=-m_{i} \vec{\omega} \times\left(\vec{\omega} \times \vec{r}_{i}^{\prime}\right)$ and Coriolis $-2 m \vec{\omega} \times \vec{v}_{i}^{\prime}$ components of inertial forces acting individually on each bead with $\vec{v}_{i}^{\prime}=\dot{\vec{r}}_{i}-\vec{\omega} \times \vec{r}_{i}$ and $X^{\prime}$ denoting a quantity in the rotating frame. For large damping $\gamma$, Eq. (1) leads to small velocities $\vec{v}_{i}^{\prime}$ such that the centrifugal component typically dominates over the Coriolis term. Hence the torque $\tau(t)$ in the rest frame can be written as

$$
\begin{aligned}
\tau(t) & =\sum_{i}^{2}\left(\vec{R}-\vec{r}_{i}\right) \times\left(\vec{r}_{i} m_{i} \omega^{2}\right)=-\frac{m}{2} \tilde{\mu} l \omega^{2}(\hat{n} \times \vec{R}) \\
& =-\frac{m}{2} \tilde{\mu} l \omega^{2} R \sin (\theta-\phi),
\end{aligned}
$$

where $\tilde{\mu} \equiv \frac{m_{1}-m_{2}}{m}$ is the scaled mass difference between the two beads, such that maximal rotation-induced torque is recovered in the limit of $|\tilde{\mu}| \rightarrow 1$. For simplicity, we consider in this work the scenario of a bottom heavy particle $\left(m_{1}>m_{2}\right)$ where the rototactic effect is maximal, such that $\tilde{\mu} \rightarrow 1$. Note that here we have parametrized the geometrical center $\vec{R}$ in the polar coordinates where $\theta$ is the positional phase angle of $\vec{R}$ (i.e., $\vec{R}=R[\cos \theta, \sin \theta]^{T}$ ) in the rest frame and is defined from the same axis that defines $\phi$. See Figs. 1(a) and 1(b) for schematics of the model microswimmer in the rotating environment.

Here we remark that a torque $\mathcal{T} \sim R \sin (\theta-\phi)$ that plays the same essential role as $\tau$ in orientating the swimmer towards the rotation center $\hat{O}$ in the present model can also be physically realized by placing a light source at the rotation center for phototactic particles [15-25] or by placing a pole of a magnetic field at $\hat{O}$ for magnetotactic swimmers [26-31].

\section{OVERDAMPED DYNAMICS}

In this section, we consider overdamped dynamics of the rototactic particle by neglecting inertial terms $(J \ddot{\phi}=0$ and $m \ddot{\vec{R}}=0)$ and any internal torque $(M=0)$ in the noise-free 
limit $[\vec{f}(t)=\vec{g}(t)=0]$ of the rest frame Eqs. (1) and (2):

$$
\begin{aligned}
\dot{\vec{R}} & =v_{0} \hat{n}+\vec{\omega} \times \vec{R}, \\
\dot{\phi} & =-\frac{m}{2} \tilde{\mu} l \omega^{2} R \sin (\theta-\phi) / \gamma_{R}+\omega .
\end{aligned}
$$

These equations include the crucial presence of the rotationinduced torque $\tau(t)$ that consistently vanishes in the limit of $l \rightarrow 0$ and directly corresponds to the limit of strong activity, where $v_{0} \gg l \omega$ (see Appendix A).

We note that, in the absence of rotation $\vec{\omega}$, the overdamped limit of this model has recently been investigated for a mathematically equivalent phototactic torque $\mathcal{T}$ in a system of phototactically driven Janus particles and found experimentally to result in nontrivial trochoidal trajectories [25], which we subsequently also recover in this section. On the other hand, the zero-torque limit of this model has been studied in [70].

Now, the radial and angular components of Eq. (5) explicitly read

$$
\begin{aligned}
\dot{R} & =v_{0} \cos (\theta-\phi), \\
\dot{\theta} & =\omega-v_{0} \sin (\theta-\phi) / R .
\end{aligned}
$$

By introducing the parameter $\kappa_{0} \equiv \frac{m}{2} \tilde{\mu} l \omega^{2} / \gamma_{R}$ and a variable corresponding to the angular difference between positional and orientational phases

$$
\alpha \equiv \theta-\phi,
$$

Eqs. (5) and (6) reduce to a system consisting of only two degrees of freedom in $\alpha$ and $R$ :

$$
\begin{gathered}
\dot{R}=v_{0} \cos \alpha, \\
\dot{\alpha}=\left(\kappa_{0} R-\frac{v_{0}}{R}\right) \sin \alpha,
\end{gathered}
$$

which, in turn, is equivalent to a single separable first order differential equation that upon solving yields the following transcendental equation:

$$
\frac{R \sin \alpha}{R_{\text {int }} \sin \alpha_{\text {int }}}=\exp \left(\frac{\kappa_{0}\left(R^{2}-R_{\text {int }}^{2}\right)}{2 v_{0}}\right)
$$

where $R_{\text {int }}$ and $\alpha_{\text {int }}$ denote initial conditions of $R$ and $\alpha$ at time $t=0$, respectively.

This system of equations involving $\alpha$ has two fixed points $\left(R_{o}^{\star}, \alpha_{o, \pm}^{\star}\right)=\left(\sqrt{v_{0} / \kappa_{0}}, \pm \pi / 2\right)$ corresponding to closed circular trajectories in the $R-\theta$ plane, and can also be seen as the set of solutions where $\dot{\theta}=\dot{\phi}$ in Eqs. (7) and (8), for which $\dot{R}=0$ can only be satisfied at a critical radial distance $R_{o}^{\star}$.

A linear perturbative expansion of (10) and (11) to first order in $\delta \alpha$ and $\delta R$ about these fixed points yields

$$
\left(\begin{array}{l}
\delta \dot{R} \\
\delta \dot{\alpha}
\end{array}\right)=\left(\begin{array}{cc}
0 & \mp 1 \\
\pm 2 \kappa_{0} & 0
\end{array}\right)\left(\begin{array}{l}
\delta R \\
\delta \alpha
\end{array}\right)
$$

of which upon diagonalization leads to the purely imaginary eigenvalue pair $\lambda_{o, \pm}= \pm \sqrt{2 \kappa_{0}} i$ with corresponding eigenvectors $\vec{x}_{o, \pm}=\frac{1}{\sqrt{2 \kappa_{0}+1}}\left[ \pm i, \sqrt{2 \kappa_{0}}\right]^{T}$, indicating that the fixed points are marginally stable, and that trajectories close to the fixed points are on center manifolds, and would thus be noncircular closed orbits in the $R-\theta$ plane.

In fact, an examination of the dynamical flows in phase space (see Fig. 2) indicates that all initial conditions where

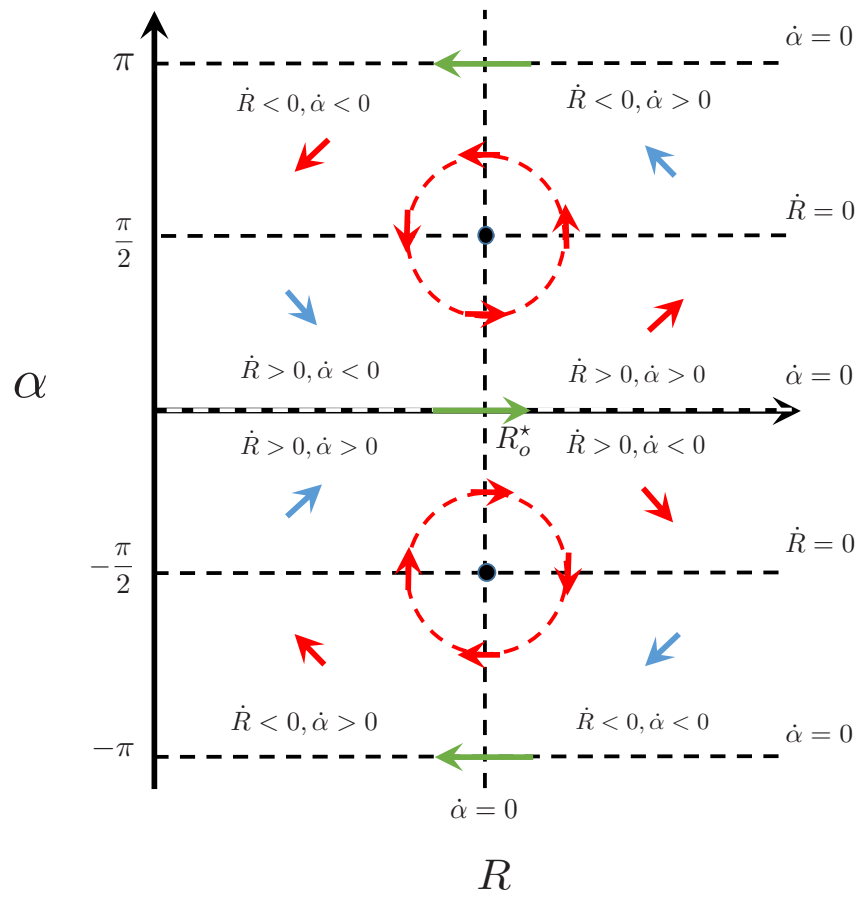

FIG. 2. Phase space of the overdamped system in $R-\alpha$ plane. Green arrows represent regions of phase space where trajectories are unstable $(R \rightarrow 0$ or $R \rightarrow \infty)$ and red arrows represent confined stable trajectories where circles in particular represent the closed stable trajectories that are predicted by the linear analysis, while blue arrows indicate regions where the stability properties are indeterminate from simply analyzing the time derivatives.

$\alpha_{\text {int }} \neq \pm \pi / 2$ result in confined trajectories where the asymptotic value of $R$ is neither infinity nor zero. This can be seen by noticing that no initial condition other than $\alpha_{\text {int }}= \pm \pi / 2$ can satisfy the transcendental solution (12) for $\alpha= \pm \pi / 2$. Similarly $a_{\text {int }}=0$ or $\pm \pi$ are the only allowed initial conditions for $R=0$.

In other words, no trajectories from the regions of phase space for which the subsequent dynamics is indeterminate, indicated by the blue arrows, will ever flow into the divergent subspace denoted by the green arrows in Fig. 2. Thus, while points in phase space where $\alpha=n \pi / 2$ results in nonconfined trajectories, these form isolated subspaces (only on a line) in phase space that are not accessible from other domains. This also implies that the sign of $\alpha$ and the rotation-induced torque is preserved throughout the entire time evolution in overdamped dynamics of this model.

It then follows that the overdamped active swimmer will always exhibit negative rototaxis in real physical systems where noise is inevitably present, since any finite perturbation away from the unstable line domains $(\alpha=n \pi)$ results in stable localized solutions. Note that this negatively rototactic behavior in the overdamped limit stems from the current context of a bottom heavy particle, i.e., $m_{1}>m_{2}$, such that $\tilde{\mu}>0$. A top heavy particle $\left(m_{1}<m_{2}\right)$ would instead result in positively rototactic behavior. Moreover, note that this argument is also based solely on the dynamical flows and is entirely general in that it is made independent of any linear 
approximation and is thus valid for arbitrarily far regions in phase space away from the fixed points.

Furthermore, we also note that the ideal rheotactic solution, where the swim orientation is perfectly aligned with the local flow, corresponds in our picture to an ideal negative rototaxis given by the fixed point solutions $\left(R_{o}^{\star}, \alpha_{o, \pm}^{\star}\right)$. On the contrary, ideal positive rototaxis occurs for the unstable line domains $\alpha= \pm \pi$, where swim orientation is always orthogonal to the direction of local flow, for which there is no equivalent counterpart in the rheotactic perspective. As such, rototaxis can be seen as a generalization of rheotactic principles.

In the remainder of this section, we focus our attention on the dynamical behavior of the linear response regime in the neighborhood of the fixed points. Explicitly the general solution of the linearized system for some initial conditions $\delta \alpha_{0}$ and $\delta R_{o}$ in the neighborhood of $R_{o}^{\star}$ and $\alpha_{o,+}^{\star}$ is given by

$$
\left(\begin{array}{l}
\delta R \\
\delta \alpha
\end{array}\right)=\frac{1}{\sqrt{2 \kappa_{0}+1}}\left[A_{+} e^{\sqrt{2 \kappa_{0}} i t}\left(\begin{array}{c}
i \\
\sqrt{2 \kappa_{0}}
\end{array}\right)+A_{-} e^{-\sqrt{2 \kappa_{0}} i t}\left(\begin{array}{c}
-i \\
\sqrt{2 \kappa_{0}}
\end{array}\right)\right],
$$

where the coefficients satisfy

$$
A_{ \pm}=\frac{\sqrt{2 \kappa_{0}+1}}{2}\left(\frac{1}{\sqrt{2 \kappa_{0}}} \delta \alpha_{0} \mp i \delta R_{0}\right) .
$$

Note that $A_{+}=A_{-}$and $A_{+}=-A_{-}$in the special cases where $\delta R_{0}=0$ and $\delta \alpha_{0}=0$, respectively. Now, by representing the complex coefficients in polar form as $A_{ \pm}=r_{A} e^{ \pm i \xi}$, we obtain an analytical expression for the dynamical trajectories of the active microswimmer in the linear regime,

$$
\left(\begin{array}{l}
\delta R \\
\delta \alpha
\end{array}\right)=\delta \tilde{R}_{\max }\left(\begin{array}{c}
-\sin \left[\left(\xi+\sqrt{2 \kappa_{0}}\right) t\right] \\
\sqrt{2 \kappa_{0}} \cos \left[\left(\xi+\sqrt{2 \kappa_{0}}\right) t\right]
\end{array}\right)
$$

where $\xi=\tan ^{-1} \sqrt{2 \kappa_{0}} \delta R_{0} / \delta \alpha_{0}$ and

$$
\delta \tilde{R}_{\max }=\sqrt{\frac{\delta \alpha_{0}^{2}}{2 \kappa_{0}}+\delta R_{0}^{2}} .
$$

This expression corresponds geometrically to ellipses centered about $\left(R_{o}^{\star}, \alpha_{o}^{\star}\right)$ in the $\alpha-R$ plane, with semimajor and minor axes given by $\delta \tilde{R}_{\max }$ and $\sqrt{2 \kappa_{0}} \delta \tilde{R}_{\max }$, respectively.

In real space, the trajectories of $\vec{R}(t)$ for dynamics near the fixed points are thus epicyclic in nature characterized by the global rotation frequency $\omega$ and the epicyclic frequency $\Omega_{\xi}=$ $\sqrt{2 \kappa_{0}}+\xi$ about the circular solution $R_{o}^{\star}$.

The geometrical intuition of this trajectory can be understood as the trace of a particle on an elliptical orbit around a point that itself is rotating about the global rotation center $\hat{O}$ in a circular fashion. This is analogous to the trace of our moon in the rest frame of the sun. Given this geometrical interpretation, $\delta \tilde{R}_{\max }$ also represents the maximum deviation in distance of a particle away from $\left(R_{o}^{\star}, \alpha_{o, \pm}^{\star}\right)$ at any point along the trajectory, thus explaining the choice of notation we have adopted.

Direct numerical integration of the equations of motion (5) and (6) also suggests that solutions are, in general, closed epicyclical periodic orbits for nonvanishing torque $\tau(t)$ resulting in rosettelike patterns in both rest and rotating frames of the system. We show representative plots of the trajectories and time evolution of relevant quantities for a specific exam-
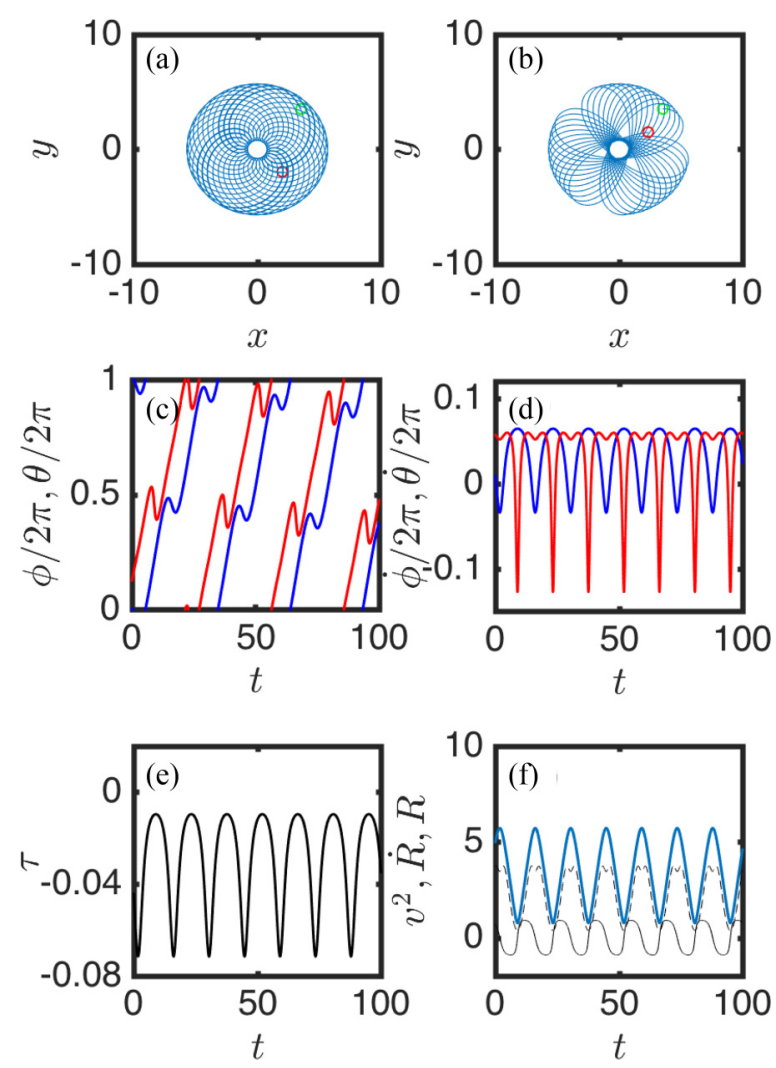

FIG. 3. Overdamped dynamics of rototactic active microswimmer. Rosette and epicyclic dynamical trajectories in the (a) rest and (b) rotating frames in real space. Green and red open circles indicate the initial and final positions of the swimmer in this particular simulation. (c) Orientational $\phi$ (blue) and positional $\theta$ (red) phase and (d) their respective phases velocities. (e) Rotation-induced torque induced. (f) Rate of change of the radial position $\dot{R}$ (black-solid line), kinetic energy $v^{2}$ (black-dashed line), and radial distance $R$ from rotation center (blue line). $\omega^{-1}, l$, and $\frac{m}{2} \tilde{\mu} l \omega^{2}$ are units of time, length, and force, respectively.

ple with initial conditions $R_{\text {int }}=5, \theta_{\text {int }}=\pi / 4, \phi_{\text {int }}=0$ (i.e., $\alpha_{\text {int }}=\pi / 4$ ) [71] (see Fig. 3).

Lastly, we also note that while the solutions obtained for the linearized system are only valid strictly in close vicinity of $\left(R_{o}^{\star}, \alpha_{o}^{\star}\right)$, we find numerically that our results are robust even for points far away from the fixed point in phase space (see Appendix B), indicating that the overdamped dynamics of the model rototactic swimmer exhibits negative rototaxis, and in general traces out localized epicycliclike trajectories.

\section{INERTIAL DYNAMICS}

In this section we extend our introduction of rototaxis to the general problem where inertia plays a significant role. While existing studies of active matter have predominantly been focused on overdamped systems [1,2] owing to the typical flow regime where a majority of microswimmers reside, there has been recent interest in understanding the role of inertia $[56,72]$. Most notably, experimental work on macroscopic synthetic self-propelled particles are found to exhibit a nontrivial inertial delay between orientation and swim directions that can significantly affect dynamical properties of steady 

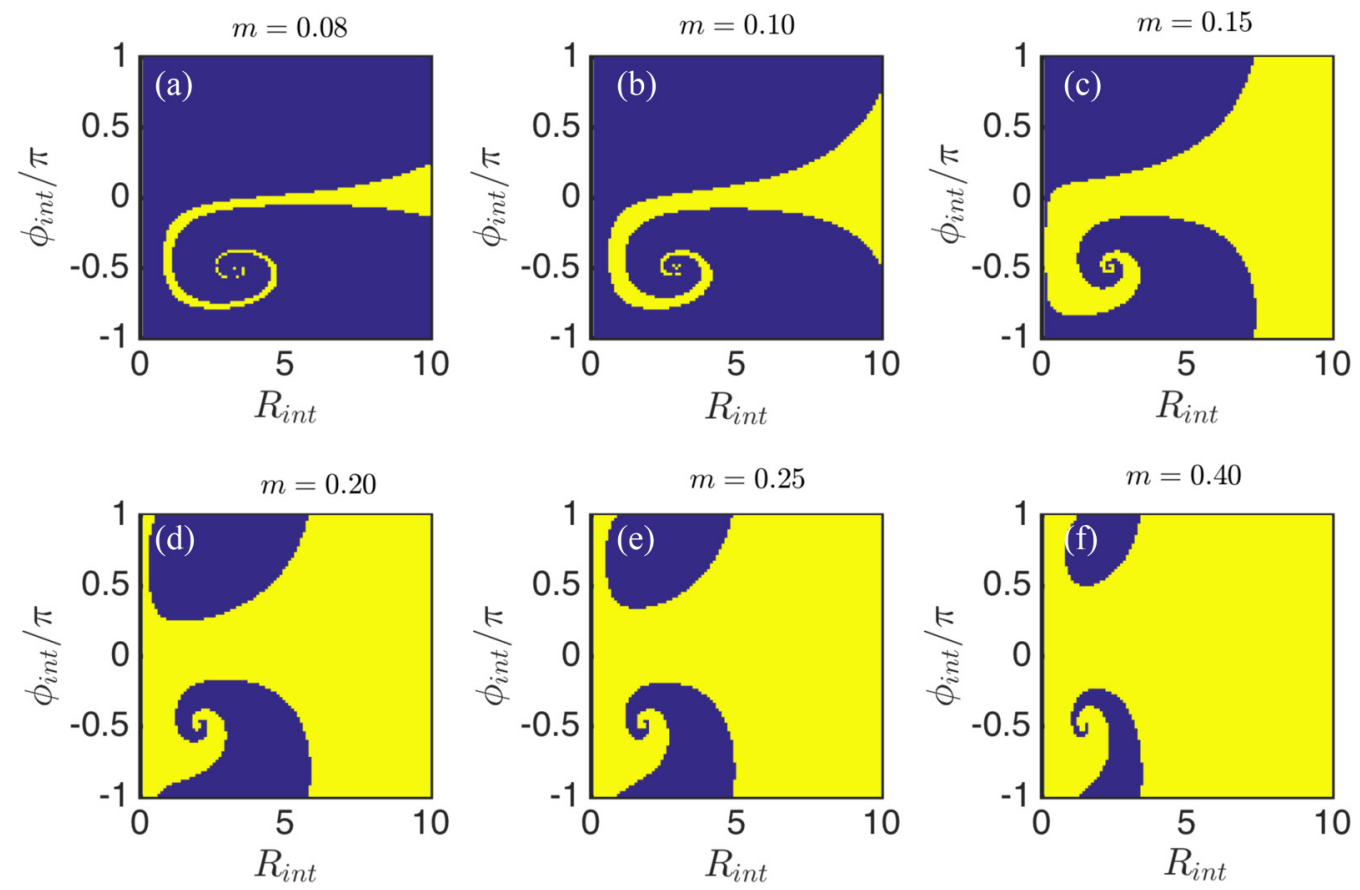

FIG. 4. Phase diagram for the inertial dynamics of rototactic particles consisting of symmetrically sized beads of total mass $m$ [(a) 0.08 , (b) 0.10 , (c) 0.15 , (d) 0.20 , (e) 0.25 , and (f) 0.40 ] for $\gamma_{R}=0.2, \gamma=0.5$, and $\tilde{\mu}=1$. Domains of negative (blue) and positive (yellow) rototaxis corresponding respectively to confined and divergent dynamical trajectories form complex nonlinear patterns over the range of initial conditions $-\pi<\phi_{\text {int }}<\pi$ and $0<R_{\text {int }}<10$.

state [54]. In addition, inertial effects can also emerge from unconventional couplings between rotational and translational degrees of freedom [55], or a consequence of the finite speed at which information can propagate in systems that exhibit collective motion [58].

Hence, to this end of elucidating the role of inertia in rototaxis, we numerically investigate Eqs. (1) and (2), under the same simplifying assumptions of $f(t)=0, g(t)=0$ and $M=0$ for a range of physical parameters and initial conditions. Note that while the form of $\tau(t)$ Eq. (4) comes from explicit consideration for the inertial forces, the equations of motion [Eqs. (1) and (2)] are defined in the noninertial frame and thus do not involve explicit inertial force terms even in the underdamped scenario. In the context of rototaxis, we are primarily interested in the $t \rightarrow \infty$ behavior of the dynamics and, in particular, its localization properties about its rotation center. Thus the natural dynamical quantity of interest is the long time or steady state behavior of the radial position along trajectory $R_{t \rightarrow \infty}$, which we characterize numerically simply by $R(t=T)$ for some finite simulation time $T$. Moreover, the difference in polynomial and exponential dependence of $R(T)$ on $T$ in scenarios of negative and positive rototaxis, respectively, leads to high numerical sensitivity and robustness that unambiguously distinguishes the two rototactic phases even for relatively short timescales $T$.

Now, without any loss of generality, we also assume that the particle is initially positioned along the positive $x$ axis such that $\theta_{\text {int }}=0$, and probe the initial condition space along the orientational phase $\phi_{\mathrm{int}}$ and radial distance $R_{\mathrm{int}}$. For simplicity, we also adopt a convention where $\gamma v_{0}, l$, and $\omega^{-1}$ are used respectively as units of force, length, and time.
In Fig. 4 we simulate [73] rototactic particles in the limit of $\tilde{\mu} \rightarrow 1$ at various values of the combined mass $m$ at values of $\gamma_{R}=0.2$ and $\gamma=0.5$ and employ an effective cutoff to the radial distance at time $T$ to distinguish domains of initial condition space where the dynamics exhibit negative (blue) or positive (yellow) rototaxis. We see that, unlike the overdamped regime, inertial dynamics of the rototactic particle is far more complex and crucially capable of supporting sizable two-dimensional domains of positive rototaxis in phase space that are robust to fluctuations in initial conditions unlike the one dimensional lines in the overdamped scenario.

Moreover, we see, from the complex tomography of the phase space across cross-sectional values of $m$, the formation of highly nontrivial patterns in the arrangement of these domains that lead to the existence of both closed finite and infinite spiral domain walls that could even appear simultaneously for a single value of $m$. This represents a high level of nonlinearity and initial condition dependence of the dynamics. Here we also note that the limit of $m \rightarrow 0$ corresponds to the overdamped dynamics considered in Sec. III and, indeed, we see that the domain size of the negative rototactic phase increases with decreasing $m$, and the presence of an asymmetry in the occurrence of negative rototaxis that favors smaller $R_{\mathrm{int}}$ and $\phi_{\text {int }}>0$, as we would expect given the result in [70] and the positively defined global rotation.

Even more surprisingly, a closer examination of the numerical trajectories reveal that, in addition to the epicyclic or rosettelike trajectories also found in the overdamped scenario, there exists circular orbits (about the global rotational center) in the steady state that correspond to stable nonlinear limit cycles [74], which we show examples of in Figs. 5(c) and 5(d). 

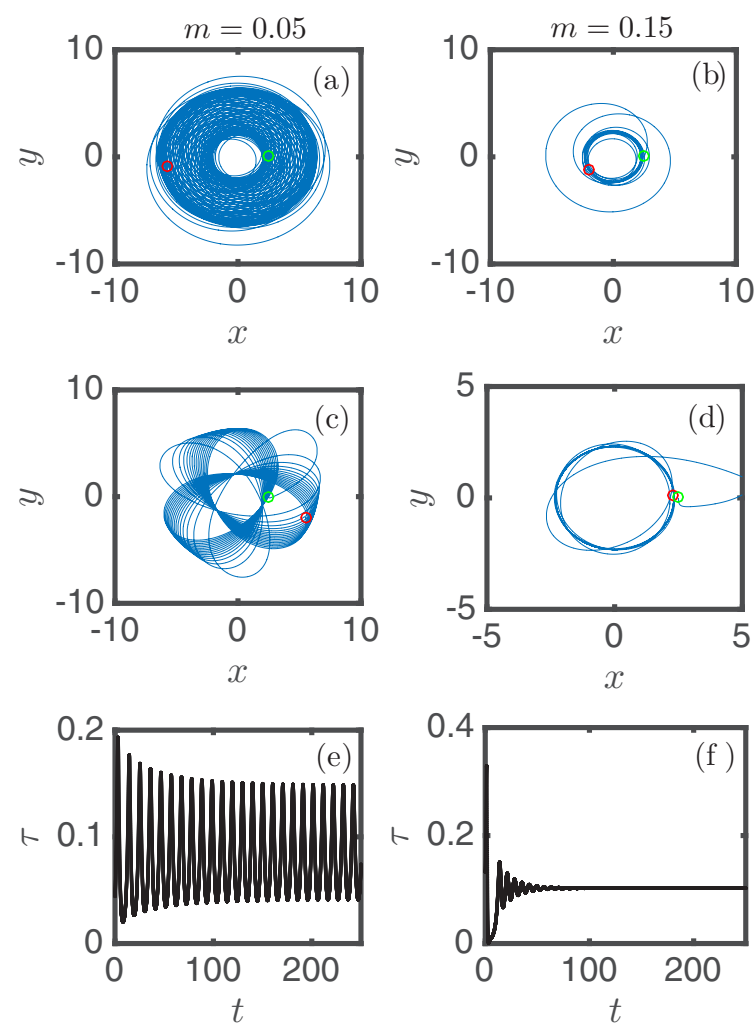

FIG. 5. Dynamical behavior of inertial systems for particles of different masses (a),(c),(e) $m=0.05$ and (b),(d),(f) $m=0.15$, for otherwise identical system parameters $\left(\gamma=0.5, \gamma_{R}=0.25\right)$ and initial conditions $\left(\theta_{\text {int }}=0, \phi_{\text {int }}=\pi / 4, R_{\text {int }}=2.5\right)$. Epicyclic orbits in the (a) rest and (c) rotating frame reminiscent of overdamped dynamics induced by a (e) periodic oscillating torque and (b) emergent nonlinear circular stable limit cycles in the (b) rest and (d) rotating frames resulting from a (f) constant torque due to phase locking. Green and red open circles represent the initial and final positions of the particle along its trajectory.

These near circular steady state orbits in the rest frame originate from a rapid damping of oscillations in the time evolution of $\tau$ [see Fig. 5(f)], such that the steady state torque is a constant [75]. Given that $\tau \sim \sin \alpha$ [4], a constant torque corresponds to stable orbital configurations where the orientational $\theta$ and positional $\phi$ angles are phase locked at some constant difference $\Delta$. This in turn represents a self driven dynamical evolution towards phase synchronization, that is often found to be emergent in various biophysical models [76-78], and is not a solution to the overdamped equations of motion (5) and (6).

In fact, notice that this emergent constant value of $\tau$ in the steady state plays the exact role of an internal torque $M$ that is often placed by hand in various models of circle swimmers that we have thus far neglected, for which the circular orbit is a known solution to the inertial dynamics under a global rotation [70]. It thus follows that the transition between overdamped epicyclic trajectories and circular limit cycles could well be understood as the onset of some linear instability of the circle solution itself.
To explore this idea in more detail, we write down the equations of motion (1) in polar form [79]

$$
\begin{gathered}
m\left[\left(\ddot{R}-R \dot{\theta}^{2}\right) \hat{e}_{R}+(2 \dot{R} \dot{\theta}+R \ddot{\theta}) \hat{e}_{\theta}\right] \\
\quad+\gamma\left[R \dot{\theta} \hat{e}_{\theta}+\dot{R} \hat{e}_{R}\right]-\gamma \omega R \hat{e}_{\theta} \\
=\gamma v_{0} \cos (\theta-\phi) \hat{e}_{R}-\gamma v_{0} \sin (\theta-\phi) \hat{e}_{\theta}, \\
\frac{m}{4} l^{2} \ddot{\phi}+\gamma_{R}(\dot{\phi}-\omega)=-\frac{m}{2} \tilde{\mu} l \omega^{2} R \sin (\theta-\phi),
\end{gathered}
$$

and assume the limit cycle (circle of radius $R_{0}$ about the center of rotation) to be a stationary solution, i.e., $\ddot{R}_{0}=$ $\dot{R}_{0}=0$ and $\ddot{\theta}_{0}=0$ to the polar equations of motion (18) and (19) satisfied by $R(t)=R_{0}, \dot{\theta}_{0}=\dot{\phi}_{0}=\omega_{0}$ and $\theta_{0}-\phi_{0} \equiv$ $\Delta$ for some steady state frequency $\omega_{0}$ and constant phase difference $\Delta$.

Substitution of this circle solution to Eq. (18) yields

$$
\begin{aligned}
-m R_{0} \omega_{0}^{2} & =\gamma v_{0} \cos \Delta, \\
\gamma R_{0}\left(\omega_{0}-\omega\right) & =-\gamma v_{0} \sin \Delta,
\end{aligned}
$$

and an expression for the radius of the limit cycle

$$
R_{0}=\frac{\gamma v_{0}}{\sqrt{m^{2} \omega_{0}^{4}+\gamma^{2}\left(\omega-\omega_{0}\right)^{2}}} \equiv b .
$$

Note that these equations imply that the periodic circular trajectory is not a solution to the equations of motion in the absence of activity (because the terms $\cos \Delta$ and $\sin \Delta$ vanish for $v_{0}=0$, such that there is in general no permissible nonzero value of $R_{0}$ ), and thus the dynamics discussed here cannot be achieved by a passive rotor.

For a system where the actual dynamics is indeed that of the circular limit cycle where $\tau$ approaches a nonzero constant, the radial position $R_{t=T}$ for a sufficiently large $T$ would be exactly equal to the radius $b$, such that $R_{t=T} / b=$ 1. However, as $m \rightarrow 0$, the system becomes increasingly overdamped, such that at some point the circle solution becomes unstable $\left(R_{t=T} / b \neq 1\right)$, although trajectories remain localized. We see this in effect in Fig. 6(a), where $R_{t=T} / b$ is strictly one at intermediate values of $m$ but discontinuously becomes "pseudorandom" at some smaller value of $m$, indicating the presence of an instability transition at some $m=m^{\star}$. Here pseudorandom refers to the behavior where $R_{t=T}$ are seemingly randomly distributed for simulations with arbitrary duration $T$, in contrast to the specific fixed value of $b$ for circular orbits. Numerical simulations suggest that $m^{\star}$ scales nonlinearly as a power law with $\gamma$, for values of $\gamma_{R}$ and $\gamma$, where it remains defined (see Appendix C).

To understand the stability properties of the circle solution at the point of transition, we now numerically find the eigenmodes of a linearized system [23] as $m \rightarrow 0$, keeping all other parameters and initial conditions constant. Specifically, we consider a (linear) perturbation $\left(\vec{R}=\vec{R}_{0}+\delta \vec{R}\right.$ and $\phi=\phi_{0}+$ $\delta \phi)$ to this stationary solution, retaining only contributions to lowest order in $\delta \vec{R}$ and $\delta \phi$. This results in a linearized system of equations in our chosen units of force, length, and time (i.e., 

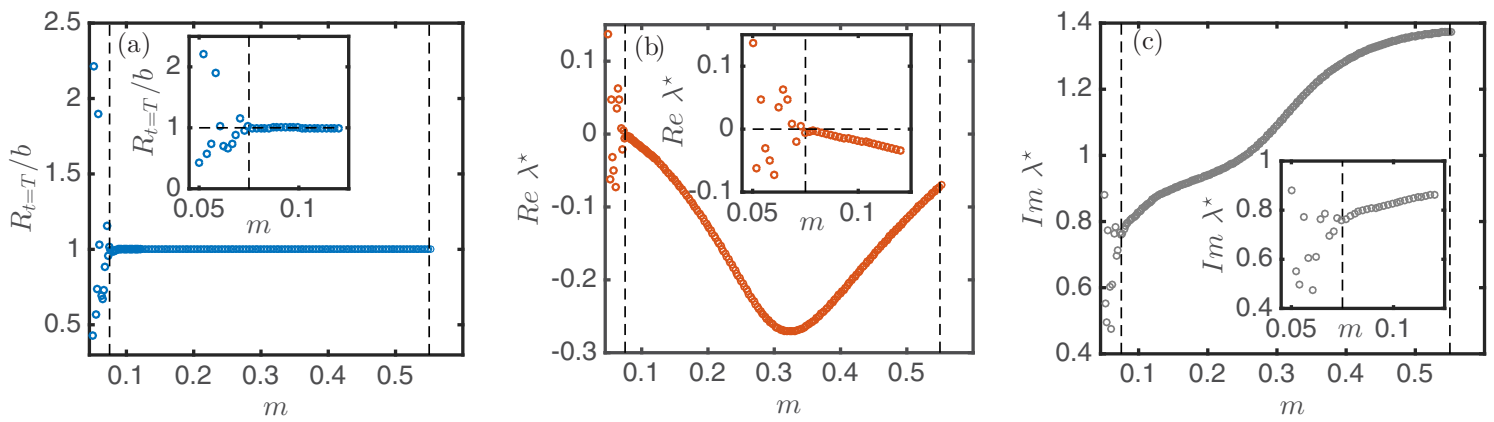

FIG. 6. (a) Ratio of the radial position $R_{t=T}$ at time $T$ to the radius of the circular ansatz $b$ as a function of particle mass $m$. (b) Real and (c) imaginary parts of the eigenvalue $\lambda^{\star}$. Inset shows magnified plots near the instability transition of the respective quantities. Note that $R_{t=T}$ and $\lambda^{\star}$ are out of their respective $y$-axis limits for larger values of $m$, as the particle exponentially escapes the center of rotation.

$\left.\gamma v_{0}=l=\omega^{-1}=1\right)$, that can be written as

$$
\left(\begin{array}{l}
\delta \ddot{R} \\
\delta \ddot{\theta} \\
\delta \ddot{\phi}
\end{array}\right)=\left(\begin{array}{ccc}
-\gamma / m & 2 R_{0} \omega_{0} & 0 \\
-2 \omega_{0} / R_{0} & -\gamma / m & 0 \\
0 & 0 & -4 \gamma_{R} / m
\end{array}\right)\left(\begin{array}{l}
\delta \dot{R} \\
\delta \dot{\theta} \\
\delta \dot{\phi}
\end{array}\right)+\left(\begin{array}{ccc}
\omega_{0}^{2} & -\sin \Delta / m & \sin \Delta / m \\
-\gamma\left(\omega_{0}-1\right) / m R_{0} & -\cos \Delta / m R_{0} & \cos \Delta / m R_{0} \\
-2 \tilde{\mu} \sin \Delta & -2 \tilde{\mu} R_{0} \cos \Delta & 2 \tilde{\mu} R_{0} \cos \Delta
\end{array}\right)\left(\begin{array}{l}
\delta R \\
\delta \theta \\
\delta \phi
\end{array}\right) .
$$

Upon obtaining the eigenmodes by diagonalizing [23] numerically, we plot in Figs. 6(b) and 6(c) the real and imaginary parts of the eigenvalue with the largest real part, which we denote as $\lambda^{\star}$, excluding the zero mode $\lambda_{0}=0$ with eigenvector $\frac{1}{\sqrt{2}}(\delta \hat{\theta}+\delta \hat{\phi})$. In particular, from Fig. 6(b), we see that the behavior of the eigenmodes is such that

$$
\lim _{m \rightarrow m^{\star}} \operatorname{Re} \lambda^{\star}=0^{-},
$$

indicating that the fixed point solution of a periodic circular limit cycle becomes unstable at the transition point $m^{\star}$, coinciding with the same point where $R_{t=T} / b$ becomes pseudorandom in Fig. 6(a). As such, the transition from overdamped epicycliclike trajectories to stable circular limit cycles is a linear instability transition. While not the focus of our current discussion, we note that our linear instability analysis also correctly identifies the instability transition to the positive rototactic phase, by exhibiting similar discontinuities in the ratio of $R_{t=T} / b$ and $R e \lambda^{\star}$ at a larger value of $m$ in Figs. 6(a) and 6(b).

\section{CONCLUSION}

In this work, we introduce the idea of rototaxis as a form of taxis for active particles in rotating environments. We present a model microswimmer capable of generating a torque that orients it towards the rotation center and will move on epicycliclike dynamical trajectories localized near its rotation center in the overdamped limit. This minimal bead model is designed to capture essential dynamics of rodlike axial propelled bacteria, but can also easily be extended modularly to more complex geometries and different swim orientations of biological or synthetic swimmers.

In the presence of inertia, the negative rototactic case sustains an additional solution that takes the form of stable circular limit cycles which undergo a linear instability transition that recovers the rosette trajectories in the overdamped limit.
More importantly, inertia also allows for initial conditions where positive rototaxis can occur, where the particle exponentially escapes the rotation center. These two rototactic phases occupy highly complex regions in phase and parameter space that dramatically increase the density of domain walls in phase space. Living near these domain walls could facilitate the switching in behavior across the two rototactic modes.

Furthermore, the rototactic principles gleaned from our model may be relevant beyond the current context of global rotations. Since the lowest order behavior of a rotation corresponds locally to shear flow, our results and understanding of rototaxis may be relevant to a wide range of microswimmer dynamics and behavior in experimentally relevant shear environments. In fact, it has been recently discovered that transport of bacteria can be heavily suppressed under linear shear $[80,81]$ and even be confined to directional motion out of the shear-flow plane for an oscillatory drive [82]. Remarkably, it has also been shown, both experimentally and theoretically, that localized cycloidlike periodic trajectories reminiscent of the rosette trajectories found in the negative rototactic phase of our model can also emerge from Poiseuille flow [83,84], suggesting that results presented for the global rotation may indeed be relevant to various shear environments beyond linear flow. These phenomena may even prove to provide evolutionary advantages to the survival of bacteria [85]. This connection to shear flow remains to be carefully examined.

As such, it would be interesting to consider rototaxis in scenarios involving more complex rotational flows with differential vorticity, alternative particle geometries [59,86,87] and their possible time dependent adaptations, and many body effects in the presence of repulsive and alignment interactions [88,89]. In even more general flow fields $\vec{u}(\vec{r})$, our methods may be useful to study general kinotaxis [alignment along $\nabla \vec{u}^{2}(\vec{r})$ towards the gradient of kinetic flow energy] and enstrotaxis [alignment along $\nabla(\nabla \times \vec{u}(\vec{r}))^{2}$ towards the gradient of the enstrophy]. 


\section{ACKNOWLEDGMENTS}

We thank E. Clement, A. Daddi-Moussa-Ider, B. Liebchen, A. M. Menzel, and C. Scholz for insightful discussions, and B. Liebchen for suggesting the term "rototaxis." H.L. is supported by the German Research Foundation DFG (project LO 418/23-1).

\section{APPENDIX A: PARAMETER REGIMES OF THE OVERDAMPED LIMIT}

In this Appendix, we discuss dynamical regimes and the corresponding range of physical parameters for which the equations of motion and results in the overdamped limit (Sec. III) are relevant.

First, we rescale the translational equation of motion (1) by introducing $\overrightarrow{R^{\prime \prime}} \equiv \vec{R} / l$ and $t^{\prime \prime}=\omega t$, corresponding to the dimensionless variables of position and time, respectively, in the limit where $f(t)=g(t)=0$ and $M=0$ :

$$
\left(\frac{m \omega}{\gamma}\right) \frac{d^{2} \vec{R}^{\prime \prime}}{d t^{\prime \prime 2}}+\frac{d \overrightarrow{R^{\prime \prime}}}{d t^{\prime \prime}}-\hat{\omega} \times \overrightarrow{R^{\prime \prime}}=\left(\frac{v_{0}}{\omega l}\right) \hat{n} .
$$

Now, since the terms $d \vec{R}^{\prime \prime} / d t^{\prime \prime}$ and $\hat{\omega} \times \vec{R}^{\prime \prime}$ are of order $\sim O\left(\vec{R}^{\prime \prime}\right)$, the inertial contribution can be neglected in the limit

$$
\frac{m \omega}{\gamma} \ll O\left(\vec{R}^{\prime \prime}\right)
$$

and thus the translational equation of motion reduces to the overdamped form (5) for regimes where

$$
O\left(\vec{R}^{\prime \prime}\right) \sim \frac{v_{0}}{l \omega} .
$$

Similarly, we obtain by substitution for the rotational degree of freedom in nondimensional form

$$
\frac{m l^{2} \omega}{4 \gamma_{R}} \ddot{\phi^{\prime \prime}}+\left(\dot{\phi^{\prime \prime}}-1\right)=\frac{m \tilde{\mu} l^{2} \omega R^{\prime \prime}}{2 \gamma_{R}} \sin \left(\theta^{\prime \prime}-\phi^{\prime \prime}\right) .
$$

Now, we see that the inertial term can be neglected even for a nonvanishing torque for

$$
\frac{m l^{2} \omega}{4 \gamma_{R}} \ll 1 \ll R^{\prime \prime} .
$$

Given that the rotational viscosity is typically $\gamma_{R} \sim O\left(\gamma l^{2}\right)$, we arrive by use of Eqs. (A2) and (A3) at the limit for the overdamped system

$$
\frac{v_{0}}{l \omega} \gg 1 .
$$

This corresponds to a physical picture of strong activity, where self-propulsion $\left(v_{0}\right)$ is significantly greater than the typical rotational motion $(l \omega)$.

\section{APPENDIX B: ROBUSTNESS OF THE LINEAR APPROXIMATION IN THE OVERDAMPED REGIME}

The dynamical trajectories and solutions of the overdamped microswimmer derived from linear response in the main text is strictly valid only for small neighborhoods around the fixed point. However, numerical simulations seem to suggest that they are in fact good approximations even

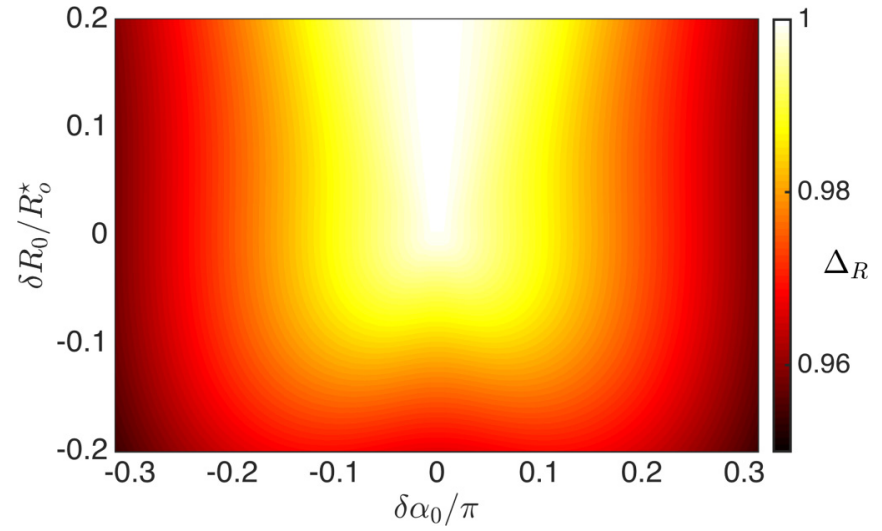

FIG. 7. Deviation $\Delta_{R} \equiv \delta \tilde{R}_{\max } / \max _{t}\left\|\vec{R}(t)-\vec{R}_{o}^{\star}(t)\right\|$ between numerics and linear approximation of the epicyclic amplitude for a range of values of the scaled initial conditions: $\delta R_{0} / R_{o}^{\star}$ and $\delta \alpha_{0} / \pi$. Parameters of the simulation are $\tilde{\mu}=1, m=1, \gamma_{R}=2$, and $\alpha_{o}^{\star}=$ $\pi / 2$, such that $R_{o}^{\star}=\sqrt{v_{0} / \kappa_{0}}=2$ in units of $\gamma v_{0}, l$, and $\omega^{-1}$ for force, length, and time, respectively.

for initial conditions considerably far from the circular ansatz.

To illustrate this, we calculate deviations between the maximum radial position (i.e., amplitude) relative to the underlying circular ansatz numerically, i.e., $\max _{t}\left|\vec{R}(t)-\vec{R}_{o}^{\star}(t)\right|$, and compare that to the analytical prediction $\delta \tilde{R}_{\max }$. We find that the actual dynamics of the particle is within 5\% of the linear approximation even for initial conditions that are up to $\pm 10 \%$ away from the fixed point, and is thus a reflection of the robustness of our results derived for the linear regime. See Fig. 7, and also note the asymmetry in the $\delta R_{0}$ axis.

\section{APPENDIX C: EXISTENCE AND SCALING BEHAVIOR OF $m^{\star}$}

Here we briefly discuss the behavior of $m^{\star}$, the critical mass at which stable closed circular orbits become unstable and instead form noncircular periodic trajectories.

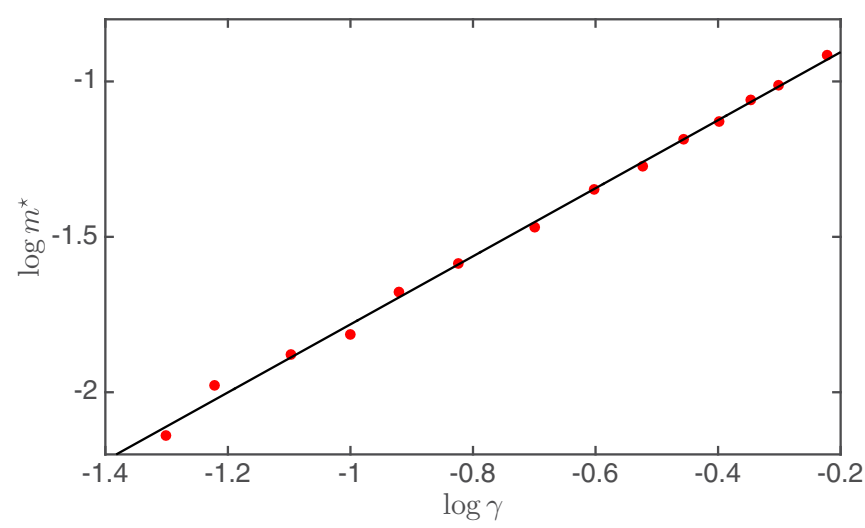

FIG. 8. $\log m^{\star}$ as a function of $\log \gamma$ for $\gamma_{R}=0.2$ (red circles). A best fit (black line) to the numercial data suggest a nonlinear power law dependence such that $m^{\star} \sim \gamma^{1.09 \pm 0.04}$. 
To begin with, we note that $m^{\star}$ may not exist for a given $\gamma$, in the limit of $\gamma_{R} \rightarrow 0$ and $\gamma_{R} \rightarrow \infty$. This behavior can be understood from physical reasoning of the respective limits. For $\gamma_{R} \rightarrow 0$, the swimmer instantaneously aligns its swim direction under the action of an external torque and is thus unable to sustain the constant phase difference between $\theta$ and $\phi$ for circular orbits to exist such that $R_{T} / b$ never approaches one and $m^{\star}$ does not exist.

Conversely, $\gamma_{R} \rightarrow \infty$ results in the inability of this swimmer to reorientate, such that stable orbits (regardless of its circular nature) cannot be formed. As such we are primarily interested in systems where $O\left(\gamma_{R}\right) \sim O\left(\gamma l^{2}\right)$, the same relative scale of the damping coefficients that we had previously assumed in Appendix A.

For finite values of $\gamma_{R}$, we investigate the behavior of $m^{\star}$ for the case of $\gamma_{R}=0.2$ that we had numerically examined in particular for $\gamma=0.5$ in the main text. Our results suggest that $m^{\star}$ scales with $\gamma$ as a power law with nonlinear index (see Fig. 8).
[1] J. Elgeti, R. G. Winkler, and G. Gompper, Physics of microswimmers-single particle motion and collective behavior, Rep. Prog. Phys. 78, 056601 (2015).

[2] C. Bechinger, R. Di Leonardo, H. Löwen, C. Reichhardt, G. Volpe, and G. Volpe, Active particles in complex and crowded environments, Rev. Mod. Phys. 88, 045006 (2016).

[3] M. C. Marchetti, J. F. Joanny, S. Ramaswamy, T. B. Liverpool, J. Prost, M. Rao, and R. A. Simha, Hydrodynamics of soft active matter, Rev. Mod. Phys. 85, 1143 (2013).

[4] D. B. Dusenbery, Living At Micro Scale (Harvard University Press, Cambridge, MA, 2009).

[5] S. J. Ebbens, Active colloids: Progress and challenges towards realising autonomous applications, Curr. Opin. Colloid Interface Sci. 21, 14 (2016)

[6] M. Eisenbach, Chemotaxis (Imperial College Press, London, 2004).

[7] Y. Hong, N. M. K. Blackman, N. D. Kopp, A. Sen, and D. Velegol, Chemotaxis of Nonbiological Colloidal Rods, Phys. Rev. Lett. 99, 178103 (2007).

[8] O. Pohl and H. Stark, Dynamic Clustering and Chemotactic Collapse of Self-Phoretic Active Particles, Phys. Rev. Lett. 112, 238303 (2014).

[9] S. Saha, R. Golestanian, and S. Ramaswamy, Clusters, asters, and collective oscillations in chemotactic colloids, Phys. Rev. E 89, 062316 (2014).

[10] B. Liebchen, D. Marenduzzo, I. Pagonabarraga, and M. E. Cates, Clustering and Pattern Formation in Chemorepulsive Active Colloids, Phys. Rev. Lett. 115, 258301 (2015).

[11] B. Liebchen, M. E. Cates, and D. Marenduzzo, Pattern formation in chemically interacting active rotors with self-propulsion, Soft Matter 12, 7259 (2016).

[12] P. Illien, R. Golestanian, and A. Sen, Fuelled motion: phoretic motility and collective behaviour of active colloids, Chem. Soc. Rev. 46, 5508 (2017).

[13] S. Henkes, M. C. Marchetti, and R. Sknepnek, Dynamical patterns in nematic active matter on a sphere, Phys. Rev. E 97, 042605 (2018).

[14] M. Paoluzzi, M. Leoni, and M. C. Marchetti, Fractal aggregation of active particles, Phys. Rev. E 98, 052603 (2018).

[15] G. Jékely, J. Colombelli, H. Hausen, K. Guy, E. Stelzer, F. Nédélec, and D. Arendt, Mechanism of phototaxis in marine zooplankton, Nature (London) 456, 395 (2008).

[16] R. R. Bennett and R. Golestanian, A steering mechanism for phototaxis in chlamydomonas, J. R. Soc. Interface 12, 20141164 (2015).
[17] A. Giometto, F. Altermatt, A. Maritan, R. Stocker, and A. Rinaldo, Generalized receptor law governs phototaxis in the phytoplankton euglena gracilis, Proc. Natl. Acad. Sci. U.S.A. 112, 7045 (2015).

[18] C. Lozano, B. ten Hagen, H. Löwen, and C. Bechinger, Phototaxis of synthetic microswimmers in optical landscapes, Nat. Commun. 7, 12828 (2016).

[19] B. Dai, J. Wang, Z. Xiong, X. Zhan, W. Dai, C.-C. Li, S. P. Feng, and J. Tang, Programmable artificial phototactic microswimmer, Nat. Nanotechnol. 11, 1087 (2016).

[20] X. Garcia, S. Rafai, and P. Peyla, Light Control of the Flow of Phototactic Microswimmer Suspensions, Phys. Rev. Lett. 110, 138106 (2013).

[21] C. Lozano and C. Bechinger, Diffusing wave paradox of phototactic particles in traveling light pulses, Nat. Commun. 10, 2495 (2019).

[22] C. Lozano, B. Liebchen, B. ten Hagen, C. Bechinger, and H. Löwen, Propagating density spikes in light-powered motilityratchets, Soft Matter 15, 5185 (2019).

[23] S. Palagi, D. P. Singh, and P. Fischer, Light-controlled micromotors and soft microrobots, Adv. Opt. Mater. 7, 1900370 (2019).

[24] W. Li, X. R. Wu, H. Qin, Z. Q. Zhao, and H. W. Liu, Lightdriven and light-guided microswimmers, Adv. Funct. Mater. 26, 3164 (2016).

[25] H. Moyses, J. Palacci, S. Sacanna, and D. G. Grier, Trochoidal trajectories of self-propelled janus particles in a diverging laser beam, Soft Matter 12, 6357 (2016).

[26] S. Klumpp and D. Faivre, Magnetotactic bacteria, Eur. Phys. J. Spec. Top. 225, 2173 (2016).

[27] J.-F. Rupprecht, N. Waisbord, C. Ybert, C. Cottin-Bizonne, and L. Bocquet, Velocity Condensation for Magnetotactic Bacteria, Phys. Rev. Lett. 116, 168101 (2016).

[28] N. Waisbord, C. T. Lefèvre, L. Bocquet, C. Ybert, and C. Cottin-Bizonne, Destabilization of a flow focused suspension of magnetotactic bacteria, Phys. Rev. Fluids 1, 053203 (2016).

[29] M. A. Fernandez-Rodriguez, F. Grillo, L. Alvarez, M. Rathlef, I. Buttinoni, G. Volpe, and L. Isa, Active colloids with positiondependent rotational diffusivity, arXiv:1911.02291.

[30] B. Vincenti, G. Ramos, M. L. Cordero, C. Douarche, R. Soto, and E. Clement, Magnetotactic bacteria in a droplet selfassemble into a rotary motor, Nat. Commun. 10, 5082 (2019).

[31] A. R. Sprenger, M. A. Fernandez-Rodriguez, L. Alvarez, L. Isa, R. Wittkowski, and H. Löwen, Active Brownian motion with orientation-dependent motility: Theory and experiments, Langmuir (2020), doi:10.1021/acs.langmuir.9b03617. 
[32] P. R. Richter, M. Schuster, M. Lebert, C. Streb, and D.-P. Haeder, Gravitaxis of euglena gracilis depends only partially on passive buoyancy, Adv. Space Res. 39, 1218 (2007).

[33] A. M. Roberts, The mechanics of gravitaxis in paramecium, J. Exp. Biol. 213, 4158 (2010).

[34] B. ten Hagen, F. Kümmel, R. Wittkowski, D. Takagi, H. Löwen, and C. Bechinger, Gravitaxis of asymmetric self-propelled colloidal particles, Nat. Commun. 5, 4829 (2014).

[35] A. I. Campbell and S. J. Ebbens, Gravitaxis in spherical janus swimming devices, Langmuir 29, 14066 (2013).

[36] A. I. Campbell, R. Wittkowski, B. ten Hagen, H. Löwen, and S. J. Ebbens, Helical paths, gravitaxis, and separation phenomena for mass-anisotropic self-propelling colloids: experiment versus theory, J. Chem. Phys. 147, 084905 (2017).

[37] K. Wolff, A. M. Hahn, and H. Stark, Sedimentation and polar order of active bottom-heavy particles, Eur. Phys. J. E 36, 43 (2013).

[38] A. Bahat, I. Tur-Kaspa, A. Gakamsky, L. C. Giojalas, H. Breitbart, and M. Eisenbach, Thermotaxis of mammalian sperm cells: a potential navigation mechanism in the female genital tract, Nat. Med. 9, 149 (2003).

[39] Y. Li, Y. Zhao, X. Huang, X. Lin, Y. Guo, D. Wang, C. Li, and C. D. Wang, Serotonin control of thermotaxis memory behavior in nematode caenorhabditis elegans, PLoS One 8, e77779 (2013).

[40] A. P. Bregulla and F. Cichos, in Optical Trapping and Optical Micromanipulation XIII, edited by K. Dholakia and G. C. Spalding (Curran Associates, Red Hook, NY, 2016).

[41] B. Liebchen, P. Monderkamp, B. ten Hagen, and H. Löwen, Viscotaxis: Microswimmer Navigation in Viscosity Gradients, Phys. Rev. Lett. 120, 208002 (2018).

[42] H. C. Fu, T. R. Powers, and R. Stocker, Bacterial rheotaxis, Proc. Natl. Acad. Sci. U.S.A. 109, 4780 (2012).

[43] A. Mathijssen, N. Figueroa-Morales, G. Junot, E. Clement, A. Lindner, and A. Zöttl, Oscillatory surface rheotaxis of swimming E. coli bacteria, Nat. Commun. 10, 3434 (2019).

[44] Q. Brosseau, F. B. Usabiaga, E. Lushi, Y. Wu, L. Ristroph, J. Zhang, M. Ward, and M. J. Shelley, Relating Rheotaxis and Hydrodynamic Actuation using Asymmetric Gold-Platinum Phoretic Rods, Phys. Rev. Lett. 123, 178004 (2019).

[45] J. Park, D.-H. Kim, H.-N. Kim, C. J. Wang, M. K. Kwak, E. Hur, K.-Y. Suh, S. S. An, and A. Levchenko, Directed migration of cancer cells guided by the graded texture of the underlying matrix, Nat. Mater. 15, 792 (2016).

[46] J. Park, D.-H. Kim, and A. Levchenko, Topotaxis: A new mechanism of directed cell migration in topographic ecm gradients, Biophys. J. 114, 1257 (2018).

[47] G. Volpe and G. Volpe, The topography of the environment alters the optimal search strategy for active particles, Proc. Natl. Acad. Sci. U.S.A. 114, 11350 (2017).

[48] K. Schakenraad, L. Ravazzano, N. Sarkar, J. A. J. Wondergem, R. M. H. Merks, and L. Giomi, Topotaxis of active Brownian particles, Phys. Rev. E 101, 032602 (2020).

[49] M. Tarama, A. M. Menzel, and H. Löwen, Deformable microswimmer in a swirl: Capturing and scattering dynamics, Phys. Rev. E 90, 032907 (2014).

[50] N. Küchler, H. Löwen, and A. M. Menzel, Getting drowned in a swirl: Deformable bead-spring model microswimmers in external flow fields, Phys. Rev. E 93, 022610 (2016).
[51] R. Supekar, V. Heinonen, K. J. Burns, and J. Dunkel, Linearly forced fluid flow on a rotating sphere, J. Fluid Mech. 892, A30 (2020).

[52] O. Akanyeti, J. Putney, Y. R. Yanagitsuru, G. V. Lauder, W. J. Stewart, and J. C. Liao, Accelerating fishes increase propulsive efficiency by modulating vortex ring geometry, Proc. Natl. Acad. Sci. U.S.A. 114, 13828 (2017).

[53] T. Viscek and A. Zafeiris, Collective motion, Phys. Rep. 517, 71 (2012).

[54] C. Scholz, S. Jahanshani, A. Ldov, and H. Löwen, Inertial delay of self-propelled particles, Nat. Commun. 9, 5156 (2018).

[55] I. Fouxon and Y. Or, Inertial self-propulsion of spherical microswimmers by rotation-translation coupling, Phys. Rev. Fluids 4, 023101 (2019).

[56] H. Löwen, Inertial effects of self-propelled particles: From active Brownian to active Langevin motion, J. Chem. Phys. 152, 040901 (2020).

[57] L. Giomi, N. Hawley-Weld, and L. Mahadevan, Swarming, swirling and stasis in sequestered bristle-bots, Proc. R. Soc. A 469, 20120637 (2013).

[58] A. Attanasi, A. Cavagna, L. Del Castello, I. Giardina, T. S. Grigera, A. Jelić, S. Melillo, L. Parisi, O. Pohl, E. Shen, and M. Viale, Information transfer and behavioural inertia in starling flocks, Nat. Phys. 10, 691 (2014).

[59] J. A. Taylor, S. R. Sichel, and N. R. Salama, Bent bacteria: a comparison of cell shape mechanisms in proteobacteria, Annu. Rev. Microbiol. 73, 457 (2019).

[60] A. Najafi and R. Golestanian, Simple swimmer at low Reynolds number: Three linked spheres, Phys. Rev. E 69, 062901 (2004).

[61] A. Daddi-Moussa-Ider, M. Lisicki, A. Mathijssen, C. Hoell, S. Goh, J. Blawzdziewicz, A. M. Menzel, and H. Löwen, State diagram of a three-sphere microswimmer in a channel, J. Phys.: Condens. Matter 30, 254004 (2018).

[62] A. Daddi-Moussa-Ider, M. Lisicki, C. Hoell, and H. Löwen, Swimming trajectories of a three-sphere microswimmer near a wall, J. Chem. Phys. 148, 134904 (2018).

[63] M. S. Rizvi, A. Farutin, and C. Misbah, Three-bead steering microswimmers, Phys. Rev. E 97, 023102 (2018).

[64] M. S. Rizvi, A. Farutin, and C. Misbah, Size and shape affect swimming of a triangular bead-spring microswimmer, Phys. Rev. E 98, 043104 (2018).

[65] S. Gonzalez and R. Soto, Directed assembly of active colloidal molecules, New J. Phys. 21, 033041 (2019).

[66] A. Sukhov, S. Ziegler, Q. Xie, O. Trosman, J. Pande, G. Grosjean, M. Hubert, N. Vandewalle, A.-S. Smith, and J. Harting, Optimal motion of triangular magnetocapillary swimmers, J. Chem. Phys. 151, 124707 (2019).

[67] G. Grosjean, M. Hubert, Y. Collard, A. Sukhov, J. Harting, A.-S. Smith, and N. Vandewalle, Capillary assemblies in a rotating magnetic field, Soft Matter 15, 9093 (2019).

[68] G. Grosjean, M. Hubert, G. Lagubeau, and N. Vandewalle, Realization of the Najafi-Golestanian microswimmer, Phys. Rev. E 94, 021101(R) (2016).

[69] F. Kümmel, B. ten Hagen, R. Wittkowski, I. Buttinoni, R. Eichhorn, G. Volpe, H. Löwen, and C. Bechinger, Circular Motion of Asymmetric Self-Propelling Particles, Phys. Rev. Lett. 110, 198302 (2013).

[70] H. Löwen, Active particles in noninertial frames: How to selfpropel on a carousel, Phys. Rev. E 99, 062608 (2019). 
[71] Simulation parameters for the overdamped dynamics are $v_{0}=$ $20, \gamma=8, \gamma_{R}=8$, where $\omega^{-1}, l$, and $\frac{m}{2} \tilde{\mu} l \omega^{2}$ are units of time, length, and force, respectively.

[72] D. Klotsa, As above, so below, and also in between: mesoscale active matter in fluids, Soft Matter 15, 8946 (2019).

[73] Numerical integration of the dynamics is performed using a fourth order Runge-Kutta method with a constant time step $\Delta t=10^{-2}$ for a duration of $T=10^{3}$. Phase determination is based on a radial cutoff distance of $R_{t=T}=5 \times 10^{2}$.

[74] J. Einarsson, J. R. Angilella, and B. Mehlig, Orientational dynamics of weakly inertial axisymmetric particles in steady viscous flows, Physica D 278, 79 (2014).

[75] Note that the swimmer could move transiently towards the center of rotation at various phases of its orbit.

[76] D. Levis, I. Pagonabarraga, and B. Liebchen, Activity induced synchronization: Mutual flocking and chiral self-sorting, Phys. Rev. Research 1, 023026 (2019).

[77] N. Uchida and R. Golestanian, Generic Conditions for Hydrodynamic Synchronization, Phys. Rev. Lett. 106, 058104 (2011).

[78] N. Uchida and R. Golestanian, Hydrodynamic synchronization between objects with cyclic rigid trajectories, Eur. Phys. J. E 35, 135 (2012).

[79] Note that the unit vector of the self-propulsion in polar coordinates are obtained by a projection of $\hat{n}$ in the Cartesian basis onto the unit vectors in polar coordinates- $\hat{n}=\cos \phi \hat{e}_{x}+$ $\sin \phi \hat{e}_{y}=\cos (\theta-\phi) \hat{e}_{R}-\sin (\theta-\phi) \hat{e}_{\theta}$.

[80] M. Borgnino, K. Gustavsson, F. De Lillo, G. Boffetta, M. Cencini, and B. Mehlig, Alignment of Nonspherical Active
Particles in Chaotic Flows, Phys. Rev. Lett. 123, 138003 (2019).

[81] R. Rusconi, J. S. Guasto, and R. Stocker, Bacterial transport suppressed by fluid shear, Nat. Phys. 10, 212 (2014).

[82] A. Hope, O. A. Croze, W. C. K. Poon, M. A. Bees, and M. D. Haw, Resonant alignment of microswimmer trajectories in oscillatory shear flows, Phys. Rev. Fluids 1, 051201(R) (2016).

[83] G. Junot, N. Figueroa-Morales, T. Darnige, A. Lindner, R. Soto, H. Auradou, and E. Clèment, Swimming bacteria in Poiseuille flow: the quest for active Bretherton-Jeffery trajectories, Europhys. Lett. 126, 44003 (2019).

[84] A. Zöttl and H. Stark, Periodic and quasiperiodic motion of an elongated microswimmer in Poiseuille flow, Eur. Phys. J. E 36, 4 (2013).

[85] G. Uppal and D. C. Vural, Shearing in flow environment promotes evolution of social behavior in microbial populations, eLife 7, e34862 (2018).

[86] R. Rusconi and R. Stocker, Microbes in flow, Curr. Opin. Microbiol. 25, 1 (2015).

[87] R. Schuech, T. Hoehfurtner, D. J. Smith, and S. Humphries, Motile curved bacteria are pareto-optimal, Proc. Natl. Acad. Sci. U.S.A. 116, 14440 (2019).

[88] J. E. Herbert-Read, A. Perna, R. P. Mann, T. M. Schaerf, D. J. T. Sumpter, and A. J. W. Ward, Inferring the rules of interaction of shoaling fish, Proc. Natl. Acad. Sci. U.S.A. 108, 18726 (2011).

[89] W. Bialek, A. Cavagna, I. Giardina, T. Mora, E. Silvestri, M. Viale, and A. M. Walczak, Statistical mechanics for natural flocks of birds, Proc. Natl. Acad. Sci. U.S.A. 109, 4786 (2012). 